\title{
Efficient Tests for Equivalence of Hidden Markov Processes and Quantum Random Walks
}

\author{
Ulrich Faigle $^{1}$ and Alexander Schönhuth ${ }^{2}$, Member IEEE \\ 1 Mathematical Institute \\ Center for Applied Computer Science \\ University of Cologne \\ Weyertal 80 \\ 50931 Köln, Germany \\ 2 Centrum Wiskunde \& Informatica \\ Science Park 123 \\ 1098 XG Amsterdam \\ as@cwi.nl
}

\begin{abstract}
While two hidden Markov process (HMP) resp. quantum random walk (QRW) parametrizations can differ from one another, the stochastic processes arising from them can be equivalent. Here a polynomialtime algorithm is presented which can determine equivalence of two HMP parametrizations $\mathcal{M}_{1}, \mathcal{M}_{2}$ resp. two QRW parametrizations $\mathcal{Q}_{1}, \mathcal{Q}_{2}$ in time $O\left(|\Sigma| \max \left(N_{1}, N_{2}\right)^{4}\right)$, where $N_{1}, N_{2}$ are the number of hidden states in $\mathcal{M}_{1}, \mathcal{M}_{2}$ resp. the dimension of the state spaces associated with $\mathcal{Q}_{1}, \mathcal{Q}_{2}$, and $\Sigma$ is the set of output symbols. Previously available algorithms for testing equivalence of HMPs were exponential in the number of hidden states. In case of QRWs, algorithms for testing equivalence had not yet been presented. The core subroutines of this algorithm can also be used to efficiently test hidden Markov processes and quantum random walks for ergodicity.
\end{abstract}

Keywords. Dimension, Discrete Random Sources, Hidden Markov Processes, Identifiability, Linearly Dependent Processes, Quantum Random Walks,

\section{Introduction}

Let a parameterized class of stochastic processes be described by a mapping

$$
\Phi: \mathcal{P} \rightarrow \mathcal{S}
$$

where $\mathcal{P}$ is the set of parameterizations and $\mathcal{S}$ is the corresponding set of stochastic processes. A stochastic process $\Phi(P)$ as induced by the parameterization $P$ is said to be identifiable iff

$$
\Phi^{-1}(\Phi(P))=\{P\}
$$

that is, iff the parameterization giving rise to it is uniquely determined. The entire class of stochastic processes $\Phi(\mathcal{P})$ is said to be identifiable iff $\Phi: \mathcal{P} \rightarrow \Phi(\mathcal{P})$ is one-toone. The equivalence problem (EP) emerges when $\Phi$ is many-to-one and is to decide 
whether two parameterizations $P_{1}, P_{2}$ are equivalent, that is $\Phi\left(P_{1}\right)=\Phi\left(P_{2}\right)$. Understanding its solutions can significantly foster understanding of the classes of stochastic processes under consideration as it usually yields insights about the class' complexity and its number of free parameters. Therefore, apart from its theoretical relevance, it is an important issue in the practice of system identification (e.g. [18]).

Hidden Markov processes (HMPs) are a class of processes which have gained widespread attention. In practical applications, for example, they have established gold standards in speech recognition and certain areas of computational biology. See e.g. [19]617] for comprehensive related literature. In an intuitive description, a hidden Markov process is governed by a Markov process which, however, cannot be observed. Observed symbols are emitted according to another set of distributions which govern the hidden, non-observed states. Since observed processes can coincide although the nonobserved processes on the hidden states can differ from one another, hidden Markov processes are non-identifiable.

For hidden Markov processes, the EP was first discussed in 1957 [3] (see also [11] for a subsequent contribution). It was formulated for finite functions of Markov chains (FFMCs), an alternative way of parametrizing hidden Markov processes where, as sets of parametrizations, the parametrizations discussed here, also referred to as hidden Markov models (HMMs) in the following, models trivially contain FFMCs. The EP for hidden Markov processes was fully solved in 1992 [13]. The corresponding algorithm is exponential in the number of hidden states and therefore impractical for larger models. See [13] also for more related work.

Quantum random walks ( $Q R W s$ ) have been introduced to quantum information theory as an analog of classical Markov sources [1]. For example, they allow emulation of Markov Chain Monte Carlo approaches on quantum computers. A collection of results has pointed out that they would be superior to their classical counterparts with respect to a variety of aspects (see e.g. [15|2[16]). However, although their mechanisms can be described in terms of elementary linear algebraic definitions, their properties are much less understood. The key element of a quantum random walk parametrization is a graph whose vertices are the observed symbols. Quantum probability distributions on the vertices are transformed by linear operations which describe the quantum mechanical concepts of evolution and measurement. It is easy to see that quantum random walks are non-identifiable. For example, any of the (infinitely many different) parametrizations with a graph of only one vertex yields the same, trivial process. The equivalence problem for quantum random walks has not been discussed before.

Beyond the work cited in [13], there is a polynomial-time solution to test equivalence of probabilistic automata [21] where HMMs can be viewed as probabilistic automata with no final probabilities [5]. The crucial difference, however, is that probabilistic automata do not give rise to stochastic processes (distributions over infinite-length sequences), but to probability distributions over the set of strings of finite length. The algorithm presented in [21] decisively depends on this and therefore does neither apply for hidden Markov processes nor for quantum random walks. Conversely, by adding a stop symbol to the set of observed symbols, any probability distribution over the set of strings of finite length can be viewed as a probability distribution over the set of infinite-length symbol sequences. This way, it can be seen that our solution also applies 
for probabilistic automata and therefore is more general than [21]'s solution.

Overall, the purpose of this work is to present a simple, polynomial-time algorithm that solves the EP for both hidden Markov processes and quantum random walks:

Theorem 1. Let $\Sigma$ be a finite set of symbols and

$$
\mathcal{M}_{X}, \mathcal{M}_{Y} \quad \text { resp. } \quad \mathcal{Q}_{X}, \mathcal{Q}_{Y}
$$

be two hidden Markov process resp. quantum random walk parametrizations giving rise to the processes $\left(X_{t}\right),\left(Y_{t}\right)$ emitting symbols from $\Sigma$. Let

$$
n_{X}, n_{Y}
$$

be the cardinalities of the set of hidden states in the hidden Markov models resp. the dimensions of the state spaces associated with the quantum random walks. Equivalence of $\left(X_{t}\right),\left(Y_{t}\right)$ can be determined in

$$
O\left(|\Sigma| \max \left\{n_{X}, n_{Y}\right\}^{4}\right)
$$

arithmetic operations.

Remark 1. Note that a polynomial-time solution for the identifiability problem for HMPs does not provide a polynomial-time solution for the graph isomorphism problem. There are both non-equivalent HMPs which act on sets of hidden states which are isomorphic as graphs (e.g. two HMPs both acting on only one hidden state which, however, have different emission probability distributions) and equivalent HMPs where underlying graphs are non-ismorphic (e.g. two HMPs, one acting on two hidden states, but emitting the symbol $a$ with probability 1 from both states and the other one acting on only one hidden state, also emitting the symbol $a$ with probability 1 , both result in the stochastic process which generates aaaa... with probability 1).

Remark 2. In [20] it was described how to test HMPs for ergodicity. Plugging the algorithm for computation of a basis (see subsection 5.1) into the generic ergodicity tests provided in [20] renders these tests efficient.

\subsection{Organization of Sections}

The core ideas of this work are tightly interconnected with the theory of finitary processes. Therefore, we start by concisely revisiting their theory in section 2 . We then introduce hidden Markov models and quantum random walk parametrizations and the mechanisms which give rise to the associated processes in section 3 In section 4 we outline how to most efficiently compute probabilities for both hidden Markov processes and quantum random walks. The algorithm and theorems behind our efficient equivalence tests are then presented in section 5. We finally outline some complementary applications of our algorithms and make some conclusive remarks. 


\section{Finitary Random Processes}

Throughout this paper, we consider discrete random processes $\left(X_{t}\right)$ that take values in the (fixed) finite alphabet $\Sigma$. We assume that the process emits the empty word $\square$ at time $t=0$. We denote the probability function $p$ of $\left(X_{t}\right)$ by

$$
p\left(a_{1} \ldots a_{t}\right):=\operatorname{Pr}\left\{X_{1}=a_{1}, \ldots, X_{t}=a_{t}\right\} \quad\left(a_{1} \ldots a_{t} \in \Sigma^{t}\right) .
$$

As usual, we set

$$
\Sigma^{*}:=\bigcup_{t \geq 0} \Sigma^{t} \quad\left(\text { with } \Sigma^{0}=\{\square\}\right)
$$

and note that $\Sigma^{*}$ is a semigroup under the concatenation $w v \in \Sigma^{s+t}$ for $w \in \Sigma^{s}$ and $v \in \Sigma^{t} .|v|=\ell$ is the length of a word $v \in \Sigma^{\ell}$.

For any $v, w \in \Sigma^{*}$, we define functions $p_{v}, p^{w}: \Sigma^{*} \rightarrow \mathbb{R}$ via

$$
p_{v}(w):=p(w v)=: p^{w}(v)
$$

and view $\mathbb{R}^{\Sigma^{*}}$ as a vector space. $p\left(v=v_{1} \ldots v_{t} \mid w=w_{1} \ldots w_{s}\right)$ generally denotes the conditional probability

$$
\begin{aligned}
p(v \mid w) & :=\operatorname{Pr}\left(X_{s+1}=v_{1}, \ldots, X_{t+s}=v_{t} \mid X_{1}=w_{1}, \ldots, X_{s}=w_{s}\right) \\
& =\left\{\begin{array}{cl}
p(v) & \text { if } w=\square \\
0 & \text { if } p(w)=0 \\
p(w v) / p(w) & \text { otherwise. }
\end{array}\right.
\end{aligned}
$$

Furthermore, the subspace

$$
\mathcal{R}(p):=\operatorname{span}\left\{p_{v} \mid v \in \Sigma^{*}\right\} \quad \text { resp. } \quad \mathcal{C}(p):=\operatorname{span}\left\{p^{w} \mid w \in \Sigma^{*}\right\}
$$

is the row space resp. column space associated with the (probability function $p$ of) the random process $\left(X_{t}\right)$.

It is easy to see that $\mathcal{R}(p)$ and $\mathcal{C}(p)$ have the same vector space dimension. So we define the dimension of $\left(X_{t}\right)$ (or its probability function $p$ ) as the parameter

$$
\operatorname{dim}\left(X_{t}\right)=\operatorname{dim}(p):=\operatorname{dim} \mathcal{R}(p)=\operatorname{dim} \mathcal{C}(p) \in \mathbb{Z}_{+} \cup\{\infty\} .
$$

For any $I, J \subseteq \Sigma^{*}$, we define the matrix

$$
P_{I J}:=[p(w v)]_{v \in I, w \in J} \in \mathbb{R}^{I \times J} .
$$

$P_{I J}$ is called generating if $\mathrm{rk}\left(P_{I J}\right)=\mathrm{rk}\left(P_{\Sigma^{*}, \Sigma^{*}}\right)(=\operatorname{dim}(p))$ and basic if it is generating and minimal among the generating $P_{I J}$, that is $|I|=|J|=\operatorname{dim}(p)$ in case of $\operatorname{dim}(p)<\infty$. In that sense, we call (in slight abuse of language) $I$ resp. $J$ a row resp. column generator/basis and the pair $(I J)$ a generator/basis for $p$.

We call a process $\left(X_{t}\right)$ finitary if it admits a (finite) basis. So the finitary processes are exactly the ones with finite dimension. 
Remark 3. The dimension of a random process is known as its minimum degree of freedom. The term finitary was introduced in [12]. Finitary processes are also called linearly dependent [14].

Theorem 2. Let $\left(X_{t}\right)$ and $\left(Y_{t}\right)$ be discrete, finitary random processes (over $\Sigma$ ) with probability functions $p$ and $q$. Let furthermore $(I J)$ be a basis for $\left(X_{t}\right)$. Then the following statements are equivalent:

(a) $p=q$.

(b) $(I, J)$ is a basis for $\left(Y_{t}\right)$ and the equalities

$$
p(v)=q(v), \quad p(w v)=q(w v) \quad \text { and } \quad p(w a v)=q(w a v)
$$

hold for all choices of $v \in I, w \in J$ and $a \in \Sigma$.

Proof. Given a basic matrix $P_{I J}$ together with the probabilities $p(v), p(w a v)$ for all $v \in I, w \in J, a \in \Sigma$, one can reconstruct $p$ via a "minimal representation" (see, e.g., [13 14 20] for details).

\section{Parametrizations and The Equivalence Problem}

\subsection{Hidden Markov Processes}

A hidden Markov process $(H M P)$ is parametrized by a tuple $\mathcal{M}=(S, E, \pi, M)$ where

1. $S=\left\{s_{1}, \ldots, s_{n}\right\}$ is a finite set of "hidden" states

2. $E=\left[e_{s a}\right] \in \mathbb{R}^{S \times \Sigma}$ is a non-negative emission probability matrix with unit row sums $\sum_{a \in \Sigma} E_{s a}=1$, (i.e. the row vectors of $E$ are probability distributions on $\Sigma$ )

3. $\pi$ is an initial probability distribution on $S$ and

4. $M=\left[m_{i j}\right] \in \mathbb{R}^{S \times S}$ is a non-negative transition probability matrix with unit row sums $\sum_{i=1}^{n} m_{i j}=1$ (i.e. the row vectors of $M$ are probability distributions on $S$

The associated process $\left(X_{t}\right)$ initially moves to a state $s \in S$ with probability $\pi_{s}$ and emits the symbol $X_{1}=a$ with probability $E_{s a}$. Then it moves from $s$ to a state $s^{\prime}$ with probability $m_{s s^{\prime}}$ and emits the symbol $X_{2}=a^{\prime}$ with probability $e_{s^{\prime} a^{\prime}}$ and so on. In the following, we also refer to a parametrization $\mathcal{M}=(S, E, \pi, M)$ as a hidden Markov model (HMM).

\subsection{Quantum Random Walks}

A quantum random walk $(Q R W)$ is parametrized by a tuple $\mathcal{Q}=\left(G, U, \psi_{0}\right)$ where

1. $G=(\Sigma, E)$ is a directed, $K$-regular graph over the alphabet $\Sigma$

2. $U: \mathbb{C}^{k} \rightarrow \mathbb{C}^{k}$ is a unitary evolution operator where $k:=|E|=K \cdot|\Sigma|$ and

3. $\psi_{0} \in \mathbb{C}^{k}$ is a wave function, that is $\left\|\psi_{0}\right\|=1$ ( $\|$.$\| the Euclidean norm).$ 
Edges are labeled by tuples $(a, x), a \in \Sigma, x \in X$ where $X$ is a finite set with $|X|=k$. Correspondingly, $\mathbb{C}^{k}$ is considered to be spanned by the orthonormal basis

$$
\left\langle\mathbf{e}_{(a, x)} \mid(a, x) \in E\right\rangle .
$$

According to [1] some more specific conditions must hold which do not affect our considerations here.

The quantum random walk $\left(X_{t}\right)$ arising from a parametrization $\mathcal{Q}=\left(G, U, \psi_{0}\right)$ proceeds by first applying the unitary operator $U$ to $\psi_{0}$ and subsequently, with probability $\sum_{x \in X}\left|\left(U \psi_{0}\right)_{(a, x)}\right|^{2}$, "collapsing" (i.e. projecting and renormalizing, which models a quantum mechanical measurement) $U \psi_{0}$ to the subspace spanned by the vectors $\mathbf{e}_{(a, x), x \in X}$ to generate the first symbol $X_{1}=a$. Collapsing $U \psi_{0}$ results in a new wave function $\psi_{1}$. Applying $U$ to $\psi_{1}$ and collapsing it, with probability $\sum_{x \in X}\left|\left(U \psi_{1}\right)_{\left(a^{\prime}, x\right)}\right|^{2}$, to the subspace spanned by $\mathbf{e}_{\left(a^{\prime}, x\right), x \in X}$ generates the next symbol $X_{2}=a^{\prime}$. Iterative application of $U$ and subsequent collapsing generates further symbols.

\subsection{The Equivalence Problem}

The equivalence problem can be framed as follows:

\section{Equivalence Problem (IP)}

Given two hidden Markov models $\mathcal{M}_{X}, \mathcal{M}_{Y}$ or two quantum random walk parametrizations $\mathcal{Q}_{X}, \mathcal{Q}_{Y}$, decide whether the associated processes $\left(X_{t}\right)$ and $\left(Y_{t}\right)$ are equivalent.

The equivalence problem can, of course, be solved in principle, in the spirit of Theorem 2 In order to efficiently solve it in practice, it suffices to be able to efficiently compute the following quantities:

(1) A basis $(I, J)$ for the finitary processes $\left(X_{t}\right),\left(Y_{t}\right)$ from their parametrizations $\mathcal{M}_{X}, \mathcal{M}_{Y}$

(2) The corresponding probabilities $p(v), p(w v), p(w a v)$ for all choices of $v \in I, w \in$ $J, a \in \Sigma$.

\section{Computing Probabilities}

We would like to point out that in the following we assume that all inputs consist of rational numbers and that each arithmetic operation can be done in constant time. This agrees with the usual conventions when treating related probabilistic concepts in terms of algorithmic complexity [19|21|5]. 


\subsection{Hidden Markov Processes}

Let now $\left(X_{t}\right)$ be a hidden Markov process with parametrization $\mathcal{M}=(S, E, \pi, M)$. Observe first that the transition matrix $M$ decomposes as $M=\sum_{a \in \Sigma} T_{a}$ into matrices $T_{a}$ with coefficients

$$
\left(T_{a}\right)_{i j}:=e_{s_{i} a} \cdot m_{i j}
$$

which reflect the probabilities to emit symbol $a$ from state $s_{i}$ and subsequently to move on to state $s_{j}$. Standard technical computations (e.g. [8]) reveal that that for any word $a_{1} \ldots a_{t} \in \Sigma^{t}$ :

$$
\begin{aligned}
p\left(a_{1} a_{2} \ldots a_{t}\right) & =p\left(a_{1} a_{2} \ldots a_{t-1}\right) p\left(a_{t} \mid a_{1} a_{2} \ldots a_{t-1}\right) \\
& =\cdots \\
& =\pi^{T} T_{a_{1}} \ldots T_{a_{t-1}} T_{a_{t}} \mathbf{1}
\end{aligned}
$$

where $\mathbf{1}=(1, \ldots, 1)^{T} \in \mathbb{R}^{S}$ is the vector of all ones.

For further reference, we use the notations

$$
T_{v}:=T_{v_{1}} T_{v_{2}} \ldots T_{v_{t-1}} T_{v_{t}} \in \mathbb{R}^{n \times n}
$$

for any $v=v_{1} \ldots v_{t} \in \Sigma^{*}$ as well as

$$
\overrightarrow{\mathbf{p}}(v):=\pi^{T} T_{v} \in \mathbb{R}^{1 \times n} \quad \text { and } \quad \overleftarrow{\mathbf{p}}(v):=T_{v} \mathbf{1} \in \mathbb{R}^{n \times 1}
$$

Remark 4. Note that computation of vectors $\overrightarrow{\mathbf{p}}(v)$ and $\overleftarrow{\mathbf{p}}(v)$ is an alternative way to describe the well-known Forward and Backward algorithm (e.g. [7]) since the entries of these two vectors can be identified with the Forward and Backward variables

$$
\begin{gathered}
\operatorname{Pr}\left(S_{s+1}=s_{i} \mid X_{1}=a_{1}, \ldots, X_{s}=a_{s}\right) \\
\text { and } \\
\operatorname{Pr}\left(S_{s+1}=s_{i} \mid X_{s+1}=a_{s+1}, \ldots, X_{s+t}=a_{s+t}\right)
\end{gathered}
$$

where $\left(S_{t}\right)$ is the (non-observable) Markov process over the hidden states $S=\left(s_{1}, \ldots, s_{n}\right)$.

\subsection{Quantum Random Walks}

The following considerations can be straightforwardly derived from standard quantum mechanical arguments, see [4] for a reference.

The State Space $\mathcal{S}^{\boldsymbol{n}}$ We write $Q^{*}$ for the adjoint of an arbitrarily sized matrix $Q \in$ $\mathbb{C}^{m \times n}$, (that is $Q_{j i}^{*}=a-i b$ if $Q_{j i}=a+i b$ where usage of $i$ as both a running index and a complex number should not lead to confusion). Let

$$
n:=k^{2}=|E|^{2} \text {. }
$$


We will consider the set of self-adjoint matrices

$$
\mathcal{S}^{n}:=\left\{Q \in \mathbb{C}^{k^{2}} \mid Q=Q^{*}\right\}
$$

in the following, which is usually referred to as state space in quantum mechanics. As usual, $\mathcal{S}^{n}$ can be viewed as an $n=k^{2}$-dimensional real-valued vector space. To illustrate this let

$$
\begin{aligned}
& \mathbf{e}_{m}:=(0, \ldots, 0, \underset{m}{1}, 0, \ldots, 0)^{T} \in \mathbb{C}^{K}, m=1, \ldots, k \text { and } \\
& \mathbf{f}_{m}:=(0, \ldots, 0, \underset{m}{i}, 0, \ldots, 0)^{T} \in \mathbb{C}^{K}, m=1, \ldots, k .
\end{aligned}
$$

The self-adjoint matrices

$$
\mathbf{E}_{m_{1} m_{2}}:=\left(\mathbf{e}_{m_{1}} \mathbf{e}_{m_{2}}^{*}+\mathbf{e}_{m_{2}} \mathbf{e}_{m_{1}}^{*}\right) \quad \text { and } \quad \mathbf{F}_{m_{1} m_{2}}:=\left(\mathbf{f}_{m_{1}} \mathbf{f}_{m_{2}}^{*}+\mathbf{f}_{m_{2}} \mathbf{f}_{m_{1}}^{*}\right)
$$

for all choices of $1 \leq m_{1}, m_{2} \leq k$ and $m_{1} \neq m_{2}$ for $\mathbf{F}_{m_{1} m_{2}}$ (since entries on the diagonal of self-adjoint matrices are real-valued) then form a canonical basis of $\mathcal{S}^{n}$ (note that $\mathbf{E}_{m_{1} m_{2}}=\mathbf{E}_{m_{2} m_{1}}, \mathbf{F}_{m_{1} m_{2}}=\mathbf{F}_{m_{2} m_{1}}$ ).

Linear Operations on $\mathcal{S}^{n}$ For a quantum random walk parametrization $\mathcal{Q}=(G=$ $\left.(\Sigma, E), U, \psi_{0}\right)$ we introduce the projection operators $(k:=|E|)$

$$
P_{a}: \mathbb{C}^{k} \longrightarrow \mathbb{C}^{k}, \psi \mapsto \sum_{(a, x), x \in X} \psi_{(a, x)} \mathbf{e}_{(a, x)}
$$

for all $a \in \Sigma$ which reflects projection of $\psi$ onto the subspace spanned by the $\mathbf{e}_{(a, x)}, x \in$ $X$. We find that

$$
T_{a}: \mathcal{S}^{n} \longrightarrow \mathcal{S}^{n}, Q \mapsto\left(P_{a} U\right) Q\left(P_{a} U\right)^{*}
$$

is an $\mathbb{R}$-linear operator acting on the state space $\mathcal{S}^{n}$. In analogy to the theory of hidden Markov models, where here the order on the letters has been reversed, we further define

$$
T_{v}:=T_{v_{t}} T_{v_{t-1}} \ldots T_{v_{2}} T_{v_{1}} \in \mathbb{R}^{n \times n}
$$

for any $v=v_{1} \ldots v_{t} \in \Sigma^{*}$.

Let now $Q_{\psi}:=\psi \psi^{*} \in \mathbb{C}^{k \times k}$ be the self-adjoint matrix being associated with a wave function $\psi \in \mathbb{C}^{k}$. We recall that, by definition of the quantum random walk $p$ with parametrization $\mathcal{Q}$, probabilities $p\left(v=v_{1} \ldots v_{t}\right)$ are computed as

$$
p\left(v=v_{1} \ldots v_{t}\right)=\left\|\left(P_{v_{t}} U\right)\left(P_{v_{t-1}}\right) \ldots\left(P_{v_{1}} U\right) \psi_{0}\right\|^{2}
$$

which can be rephrased as $\left(Q_{\psi_{0}}:=\psi_{0} \psi_{0}^{*}\right.$ and tr is the linear trace functional, that is the sum of the diagonal entries)

$$
p\left(v=v_{1} \ldots v_{t}\right)=\operatorname{tr} T_{v_{t}} \ldots T_{v_{1}} Q_{\psi_{0}}
$$

which yields that probabilities $p(v)$ can be computed by iterative application of multiplying $n \times n$-matrices with $n$-dimensional vectors where we recall that $Q_{\psi_{0}}$ can be taken as an element of the $n$-dimensional vector space $\mathcal{S}^{n}$. Note that $T_{v}$ acts on $Q_{\psi_{0}}$ in the sense of $\mathcal{S}^{n}$ whereas the trace functional treats $T_{v} Q_{\psi_{0}}$ as a matrix. 
Forward and Backward Algorithm Note that application of the trace functional can be rephrased as

$$
\operatorname{tr} Q=E \cdot Q \in \mathbb{R} \quad \text { where } \quad E:=\sum_{i=1}^{k} \mathbf{e}_{i} \mathbf{e}_{i}^{*}
$$

and, on the right hand side, both $E$ and $Q$ are taken as elements of $\mathcal{S}^{n}$, i.e. as $n$ dimensional vectors. Using this, we define

$$
\overrightarrow{\mathbf{p}}(v):=T_{v} Q_{\psi_{0}} \in \mathcal{S}^{n} \subset \mathbb{C}^{n^{2}} \quad \text { and } \quad \overleftarrow{\mathbf{p}}(v):=E T_{v} \in \mathbb{C}^{n^{2}}
$$

Computation of $\overrightarrow{\mathbf{p}}(v)$ and $\overleftarrow{\mathbf{p}}(v)$ can be taken as performing a quantum random walk version of the Forward and the Backward algorithm. Correspondingly, entries of $\overrightarrow{\mathbf{p}}(v)$ and $\overleftarrow{\mathbf{p}}(v)$ reflect Forward and Backward variables.

\subsection{Runtimes}

Since the multiplication of an $(n \times n)$-matrix with a vector can be done in $O\left(n^{2}\right)$ arithmetic operations, the previous considerations let us conclude:

Lemma 1. Given $\mathcal{M}$ or $\mathcal{Q}$ let $n$ be the number of hidden states $|S|$ resp. the dimension of the state space $\mathcal{S}^{n}$ associated with $\mathcal{Q}$ and p be the probability function of $\mathcal{M}$ or $\mathcal{Q}$.

1. For any $v \in \Sigma^{*}$

$$
\overrightarrow{\mathbf{p}}(v), \overleftarrow{\mathbf{p}}(v) \quad \text { and } \quad p(v)
$$

can be computed in $O\left(|v| n^{2}\right)$ arithmetic operations.

2. Upon computation of $\overrightarrow{\mathbf{p}}(w)$ computation of all

$$
p(w a) \text { and } \overrightarrow{\mathbf{p}}(w a)
$$

requires $O\left(|\Sigma| n^{2}\right)$ arithmetic operations.

3. Upon computation of $\overleftarrow{\mathbf{p}}(v)$ computation of all

$$
p(a v) \text { and } \overleftarrow{\mathbf{p}}(a v)
$$

requires $O\left(|\Sigma| n^{2}\right)$ arithmetic operations.

4. Upon computation of $\overrightarrow{\mathbf{p}}(w)$ and $\overleftarrow{\mathbf{p}}(v)$ computation of all

$$
p(w a v)
$$

requires $O\left(|\Sigma| n^{2}\right)$ arithmetic operations.

For hidden Markov models $\mathcal{M}$ this actually reflects well-known results on computation of Forward/Backward variables.

\section{Equivalence Tests}

In this section, we describe how to efficiently test two hidden Markov processes or quantum random walks $\left(X_{t}\right)$ and $\left(Y_{t}\right)$ for equivalence. We recall that a generic strategy has been established by theorem 2 Our solution proceeds according to this strategy. 


\subsection{Computation of a Basis}

We will now show how to compute a basis $(I J)$ in runtime $O\left(|\Sigma| n^{4}\right)$ for a hidden Markov process resp. a quantum random walk $p$. Therefore, assume for now that $g_{1}, \ldots, g_{n}$ : $\Sigma^{*} \rightarrow \mathbb{R}$ are probability functions the probabilities of which can be computed in the style of hidden Markov processes resp. quantum random walks and which generate the column space of $p$, i.e.,

$$
\mathcal{C}(p) \subset \operatorname{span}\left\{g_{1}, \ldots, g_{n}\right\} .
$$

Given $g_{1}, \ldots, g_{n}$, computation of a basis $(I J)$ proceeds in three steps the first two of which are analagous and the third of which is a simple procedure.

1. Compute a row generator $I$.

2. Compute a column basis $J$.

3. Reduce $I$ to a row basis.

While steps 1 and 2 both require runtime $O\left(|\Sigma| n^{4}\right)$, step 3 requires $O\left(n^{4}\right)$ which overall evaluates as $O\left(|\Sigma| n^{4}\right)$ runtime required for computation of a basis.

We discuss the steps in the following paragraphs. In a subsequent subsection, we show how to obtain suitable $g_{1}, \ldots, g_{n}$ for both hidden Markov models and quantum random walks.

Step 1: Computation of a row generator I Consider the following algorithm.

\section{Algorithm 1}

1: Define $\mathbf{g}(v)=\left(g_{1}(v), \ldots, g_{n}(v)\right) \in \mathbb{R}^{n}$.

2: $I \leftarrow\{\square\}, B_{\text {row }} \leftarrow\{\boldsymbol{g}(\square)\}, C_{\text {row }} \leftarrow \Sigma$.

3: while $C_{\text {row }} \neq \emptyset$ do

4: $\quad$ Choose $v \in C_{\text {row }}$.

5: $\quad$ if $\mathbf{g}(v)$ is linearly independent of $B_{\text {row }}$ then

6: $\quad I \leftarrow I \cup\{v\}, B_{\text {row }} \leftarrow B_{\text {row }} \cup\{\mathbf{g}(v)\}$

7: $\quad$ end if

$$
C_{\text {row }} \leftarrow C_{\text {row }} \cup\{a v \mid a \in \Sigma\}
$$

8: end while

9: output $I$.

Proposition 1. Let $I \subseteq \Sigma^{*}$ be the output of Algorithm $\square$ Then one has

$$
\mathcal{R}(p)=\operatorname{span}\left\{p_{v} \mid v \in I\right\} \quad \text { and } \quad \operatorname{dim}\left(X_{t}\right) \leq|I|
$$

where

$$
\mathcal{C}(p)=\operatorname{span}\left\{g_{1}, \ldots, g_{n}\right\} \quad \Rightarrow \quad \operatorname{dim}\left(X_{t}\right)=|I| .
$$

Furthermore,

(i) The algorithm terminates after at most $|\Sigma| \cdot n$ iterations. 
(ii) Each iteration requires $O\left(n^{3}\right)$ arithmetic operations where at most $n$ iterations need additional $O\left(|\Sigma| n^{3}\right)$ operations.

Proof. Ad $(i)$ : Because the $n$-dimensional vectors in $B_{\text {row }}$ are independent $\left|B_{\text {row }}\right| \leq$ $n$ and $|I| \leq n$ follow immediately. Since at most $\Sigma$ words are added to $C_{\text {row }}$ upon discovery of an $n$-dimensional vector which is linearly independent of those in $B_{\text {row }}$, we have $\left|C_{\text {row }}\right| \leq|\Sigma| \cdot n$ and hence at most $|\Sigma| \cdot n$ iterations.

Ad (ii): In each iteration, we perform a test for linear independency of at most $n$ vectors of dimension $n$ which requires at most $O\left(n^{3}\right)$ arithmetic operations [10]. In the at most $n$ cases where $\mathbf{g}(v)$ is linearly independent of $B_{\text {row }}$, we proceed by computing

$$
\left(g_{1}(a v), \ldots, g_{n}(a v)\right) \quad \text { and } \quad\left(\overleftarrow{\mathrm{g}_{1}}(a v), \ldots, \overleftarrow{\mathrm{g}_{n}}(a v)\right)
$$

for all $a \in \Sigma$ where $\left(\overleftarrow{\mathrm{g}_{1}}(v), \ldots, \overleftarrow{\mathrm{g}_{n}}(v)\right)$ are available from an iteration before (note that $g_{i}(\square)=1, \overleftarrow{\mathrm{g}_{i}}(\square)=(1, \ldots, 1)$ in the first iteration). Due to lemma 1 (35), this requires $O\left(|\Sigma| \cdot n^{3}\right)$ operations.

To prove $\left(\underline{38}\right.$, let $w_{0} \in \Sigma^{*}$ be arbitrary and suppose

$$
p_{w_{0}} \notin \operatorname{span}\left\{p_{v} \mid v \in I\right\} .
$$

Since $\mathcal{C}(p) \subset \operatorname{span}\left\{g_{1}, \ldots, g_{n}\right\}$, plugging $w=\square$ into lemma2 2 below implies

$$
\mathbf{g}\left(w_{0}\right) \notin \operatorname{span}\{\mathbf{g}(v) \mid v \in I\} .
$$

We will derive a contradiction. Indeed, the algorithm can only miss $w_{0}$ if $w_{0}$ had never been collected into $C_{\text {row }}$ in step 6 . This happens only in case that there is a $v_{0} \in \Sigma^{*}$ such that

$$
w_{0}=w v_{0}
$$

holds for some $w \in \Sigma^{*}$ and $\mathbf{g}\left(v_{0}\right)$ had been found to be linearly dependent of $[\mathbf{g}(v)]_{v \in I}$. Lemma 2 below then states that in such a case $p_{w_{0}} \in \operatorname{span}\left\{p_{w v} \mid v \in I\right\}$ holds and it remains to show that for each $w \in \Sigma^{*}$ and $v \in I$

$$
p_{w v} \in \operatorname{span}\left\{p_{v} \mid v \in I\right\} .
$$

This follows by induction on the length $|w|$ of $w$ from the following arguments. For each $w \in \Sigma^{*}$ we define a linear operator $\sigma_{w}$ on $\mathcal{R}(p)$ through

$$
\sigma_{w} p_{v}=p_{w v}
$$

By design of the update rule for $C_{\text {row }}$ in step 6 of algorithm 1 we immediately see that

$$
\mathbf{g}(a v) \in \operatorname{span}\{\mathbf{g}(v) \mid v \in I\}
$$

for all $a \in \Sigma$, hence by plugging $v_{0}=a v$ and $w=\square$ into lemma 2 , we obtain $p_{a v} \in \operatorname{span}\left\{p_{v} \mid v \in I\right\}$ that is

$$
\sigma_{a}\left(\operatorname{span}\left\{p_{v} \mid v \in I\right\}\right) \subset \operatorname{span}\left\{p_{v} \mid v \in I\right\}
$$


for all $a \in \Sigma$. Inductively, by observing that $\sigma_{w=w_{1} \ldots w_{t}}=\sigma_{w_{1}} \circ \ldots \circ \sigma_{w_{t}}$,

$$
\sigma_{w}\left(\operatorname{span}\left\{p_{v} \mid v \in I\right\} \subset \operatorname{span}\left\{p_{v} \mid v \in I\right\}\right.
$$

and thereby (44).

To see (39) let $\operatorname{dim}\left(X_{t}\right)<|I|$. Since $|I|=\left|B_{\text {row }}\right|$ we obtain that

$$
\operatorname{dim} \mathcal{C}(p)=\operatorname{dim}\left(X_{t}\right)<\left|B_{\text {row }}\right| \leq \operatorname{dim} \operatorname{span}\left\{g_{1}, \ldots, g_{n}\right\}
$$

hence $\mathcal{C}(p) \subsetneq \operatorname{span}\left\{g_{1}, \ldots, g_{n}\right\}$.

Lemma 2. Let $g_{1}, \ldots, g_{n}: \Sigma^{*} \rightarrow \mathbb{R}$ be such such that $\mathcal{C}(p) \subseteq \operatorname{span}\left\{g_{1}, \ldots, g_{n}\right\}$ and let $v_{0}, v_{1}, \ldots, v_{m} \in \Sigma^{*}$ be such that

$$
\left(g_{1}\left(v_{0}\right), \ldots, g_{n}\left(v_{0}\right)\right) \in \operatorname{span}\left\{\left(g_{1}\left(v_{j}\right), \ldots, g_{n}\left(v_{j}\right)\right) \mid j=1, \ldots, m\right\} \subseteq \mathbb{R}^{n} .
$$

Then one has for every $w \in \Sigma^{*}$ :

$$
p_{w v_{0}} \in \operatorname{span}\left\{p_{w v_{j}} \mid j=1, \ldots, m\right\} \subseteq \mathbb{R}^{n} .
$$

The analogous statement holds for the row space $\mathcal{R}(p)$.

Proof. By our hypothesis, there are scalars $\beta_{1}, \ldots, \beta_{m} \in \mathbb{R}$ such that

$$
\left(g_{1}\left(v_{0}\right), \ldots, g_{n}\left(v_{0}\right)\right)=\sum_{j=1}^{m} \beta_{j}\left(g_{1}\left(v_{j}\right), \ldots, g_{n}\left(v_{j}\right)\right) .
$$

Let $u \in \Sigma^{*}$ be arbitrary. Again by our hypothesis, there are scalars $\alpha_{i}, i=1, \ldots, n \in \mathbb{R}$ such that

$$
p_{u}=\sum_{i=1}^{n} \alpha_{i} g_{i} .
$$

We now compute

$$
\begin{aligned}
p_{u}\left(v_{0}\right) & \stackrel{53}{=} \sum_{i=1}^{n} \alpha_{i} g_{i}\left(v_{0}\right) \\
& \stackrel{52}{=} \sum_{i=1}^{n} \alpha_{i} \sum_{j=1}^{m} \beta_{j} g_{i}\left(v_{j}\right)=\sum_{j=1}^{m} \beta_{j} \sum_{i=1}^{n} \alpha_{i} g_{i}\left(v_{j}\right) \\
& \stackrel{53}{=} \sum_{j=1}^{m} \beta_{j} g_{u}\left(v_{j}\right)=\sum_{j=1}^{m} \beta_{j} p\left(u v_{j}\right)=\sum_{j=1}^{m} \beta_{j} p_{v_{j}}(u) .
\end{aligned}
$$

Since the $\beta_{j}$ had been determined independently of $u$, we thus conclude

$$
p_{v_{0}}=\sum_{j=1}^{m} \beta_{j} p_{v_{j}} .
$$


Let $\sigma_{w}$ be the linear operator on $\mathcal{R}(p)$ with the property

$$
\sigma_{w} p_{v}=p_{w v}
$$

Application of $\sigma_{w}$ to (54) then shows

$$
p_{v_{0} w}=\sigma^{w}\left(p_{v_{0}}\right)=\sum_{j=1}^{m} \beta_{j} \sigma_{w}\left(p_{v_{j}}\right)=\sum_{j=1}^{m} \beta_{j} p_{v_{j} w},
$$

which implies (51).

Step 2: Computation of a column basis $\boldsymbol{J}$ Having obtained the row generator $I \subseteq \Sigma^{*}$ in the step before, that is $\operatorname{dim}\left(X_{t}\right) \leq|I|$ and

$$
\mathcal{R}(p)=\operatorname{span}\left\{p_{v} \mid v \in I\right\},
$$

we can now use these functions $p_{v}$ as an input for an algorithm which is analogous to that for computing the row generator $I$.

\section{Algorithm 2}

1: Define $\boldsymbol{q}(w):=\left(p_{v}(w)=p(w v), v \in I\right) \in \mathbb{R}^{|I|}$.

2: $J \leftarrow\{\square\}, B_{\text {col }} \leftarrow\left\{\boldsymbol{q}_{w}(\square)\right\}, C_{\text {col }} \leftarrow \Sigma$

3: while $C_{\text {row }} \neq \emptyset$ do

4: $\quad$ Choose $w \in C_{\text {col }}$.

5: $\quad$ if $\boldsymbol{q}(w)$ is linearly independent of $B_{\text {col }}$ then

6: $\quad A_{\text {col }} \leftarrow A_{\text {col }} \cup\{w\}, B_{\text {col }} \leftarrow B_{\text {col }} \cup\{q(w)\}$

$$
C_{c o l} \leftarrow C_{c o l} \cup\{w a \mid a \in \Sigma\}
$$

7: $\quad$ end if

8: end while

9: output $J$

While this routine is, in essence, analogous to algorithm 1, there is one difference to be observed: Here $C_{c o l}$ gets augmented by joining $w a$ whereas $C_{\text {row }}$, in algorithm 1 was augmented by joining $a v$. This asymmetry is due to that one obtains an equivalently asymmetric statement in lemma 2 when rephrasing it for $\mathcal{R}(p)$ instead of $\mathcal{C}(p)$. As a consequence, application of (34) instead of (35) in lemma 1 is needed.

We obtain that

$$
P_{I J}=[p(w v)]_{v \in I, w \in J}
$$

is a generator for $\left(X_{t}\right)$. Since $\mathcal{R}(p)=\operatorname{span}\left\{p_{v} \mid v \in I\right\}$, by applying [39, we see that

$$
|J|=\operatorname{dim}\left(X_{t}\right) .
$$

Hence $J$ is a genuine column basis. We recall that this was not necessarily the case for $I$ which can happen to occur in the case $\mathcal{C}(p) \subsetneq \operatorname{span}\left\{g_{1}, \ldots, g_{n}\right\}$. 
All $p(w a v), v \in I, w \in J, a \in \Sigma$ can be obtained in runtime $O\left(|\Sigma| \cdot n^{4}\right)$ through application of (36) in lemma 1 making use of the $\overrightarrow{\mathbf{p}}(w), \overleftarrow{\mathbf{p}}(v)$ which were computed when executing the algorithms 1, 2,

We conclude: all necessary quantities can be obtained through $O\left(|\Sigma| \cdot n^{4}\right)$ arithmetic operations.

Step 3: Making $I$ a basis This step is simple: one removes $v$ from $I$ where $p(w v), w \in$ $J$ is linearly dependent in $P_{I J}$. This reduces the possibly too large set $I$ to a row basis and finally yields a basis $(I J)$ for $\left(X_{t}\right)$. This requires at most $n$ linear independence tests of $n$-dimensional vectors hence $O\left(n^{4}\right)$ runtime [10].

\subsection{Generating sets}

Let us call a set $\left\{g_{1}, \ldots, g_{n}\right\}$ of functions $g_{i}$ as in the previous section a set of generators for the column space $\mathcal{C}(p)$ of the hidden Markov process resp. quantum random walk $\left(X_{t}\right)$.

We can get sets of generators as follows for which probabilities $g_{i}(v)$ can be computed in the style of hidden Markov processes resp. quantum random walks as follows.

Hidden Markov Processes Given a hidden Markov model $\mathcal{M}=(S, E, \pi, M)$, consider the hidden Markov models $\mathcal{M}_{i}=\left(S, X, \mathbf{e}_{i}, M\right)$, where $\mathbf{e}_{i}$ is the $i$ th unit vector in $\mathbb{R}^{S}$. One now takes

$$
g_{i}(v)=\mathbf{e}_{i}^{T} T_{v} \mathbf{1} \quad(i=1, \ldots, n)
$$

Quantum Random Walks For a quantum random walk, as parametrized through a self-adjoint matrix $Q_{\psi_{0}}$ and linear operators $T_{v}, v \in \Sigma^{*}$ (acting on the state space see subsection 4.2), we see that

$$
g_{i}(v)=\operatorname{tr} T_{v} Q_{i} \quad(i=1, \ldots, n)
$$

where the $Q_{i}$ comprise all of the state space basis members $\mathbf{E}_{m_{1} m_{2}}, \mathbf{F}_{m_{1} m_{2}}($ see $(25)$ ).

\subsection{Summary}

Theorem 2 yields the following procedure as an efficient test for equivalence of processes $\left(X_{t}\right)$ and $\left(Y_{t}\right)$, :

1. Compute a basis for both $\left(X_{t}\right)$ and $\left(Y_{t}\right)$.

2. If $\operatorname{dim}\left(X_{t}\right) \neq \operatorname{dim}\left(Y_{t}\right)$ return not equivalent.

3. If $\operatorname{dim}\left(X_{t}\right)=\operatorname{dim}\left(Y_{t}\right)$, perform equality tests from (13).

4. Output equivalent if all of them apply and not equivalent if not. 
According to the above considerations, Step 1 can be performed in $O\left(|\Sigma| n^{4}\right)$ runtime where

$$
n=\max \left\{n_{X}, n_{Y}\right\}
$$

and $n_{X}, n_{Y}$, in case of hidden Markov processes $\left(X_{t}\right),\left(Y_{t}\right)$, are the numbers of hidden states and in case of quantum random walks $\left(X_{t}\right),\left(Y_{t}\right)$ are the dimensions of the associated state spaces. For step 2 we recall that all strings participating in the bases, as computed through algorithms 112, emerge as extensions of basis strings obtained in an earlier iterations. Application of $34|35| 36$ ) from lemma 1 then yields that all of the equality tests can be equally performed in $O\left(|\Sigma| n^{4}\right)$ arithmetic operations. (62).

These insights can be condensed into the following main theorem where $n$ as in

Theorem 3. The equivalence problem can be algorithmically solved for both hidden Markov processes and quantum random walks in $O\left(|\Sigma| n^{4}\right)$ arithmetic operations. $\diamond$

Probabilistic Automata Our solution can be straightforwardly adapted to determine equivalence of probabilistic automata which we will describe in the following. It can therefore be viewed as more general than the main result obtained in [21]. The main difference one has to keep in mind is that probabilistic automata induce probability distributions on the (countable) set of strings $\Sigma^{*}$ whereas HMMs give rise to stochastic processes, in other words to probability distributions on the (uncountably infinite) set of sequences $\Sigma^{\mathbb{N}}$. in case of probabilistic automata equivalence then translates to equality of the associated probability distributions on $\Sigma^{*}$. The following notations are adopted from [21].

Corollary 1. Let $\mathcal{A}_{1}=\left(S_{1}, \Sigma, M_{1}, \pi_{1}, F_{1}\right), \mathcal{A}_{2}=\left(S_{2}, \Sigma, M_{2}, \pi_{2}, F_{2}\right)$ be two probabilistic automata where $N_{1}=\left|S_{1}\right|, N_{2}=\left|S_{2}\right|$. Then equivalence of $\mathcal{A}_{1}, \mathcal{A}_{2}$ can be determined in $O\left((|\Sigma|+1) N^{4}\right)$ where $N=\max \left(N_{1}, N_{2}\right)$.

Proof. By adding a special symbol $\$$ to $\Sigma$ which is emitted from the final states with probability 1 the automata $\mathcal{A}_{1}, \mathcal{A}_{2}$ can be transformed into probabilistic automata with no final probabilities $\overline{\mathcal{A}}_{1}, \overline{\mathcal{A}}_{2}$. Let $p_{\overline{\mathcal{A}}_{1}}, p_{\overline{\mathcal{A}}_{2}}$ be the resulting stochastic processes. According to [5], lemmata $3-5$, proposition 8 , probabilistic automata with no final probabilities can be can be viewed as HMMs $\mathcal{M}_{1}, \mathcal{M}_{2}$ which translates to that for each $v \in \Sigma^{*}$

$$
p_{\mathcal{A}_{1}}(v)=p_{\mathcal{M}_{1}}(v) \quad \text { and } \quad p_{\mathcal{A}_{2}}(v)=p_{\mathcal{M}_{2}}(v) .
$$

Note that the transformation from $\mathcal{A}_{1,2}$ to $\mathcal{M}_{1,2}$ requires only constant time. Applying theorem 1 to $\mathcal{M}_{1}, \mathcal{M}_{2}$ yields the result.

In short, corollary scales down the runtime $O\left(\left(N_{1}+N_{2}\right)^{4}\right)$ (the size of the alphabet $|\Sigma|$ is not discussed in [21]) to $O\left(\left(\max \left(N_{1}, N_{2}\right)\right)^{4}\right)$. 
Ergodicity Tests In [20], a generic algorithmic strategy for testing ergodicity of hidden Markov processes was described, where overall efficiency hinged on computation of a basis of the tested hidden Markov processes. The algorithms described above resolve this issue. Hence ergodicity of hidden Markov processes can be efficiently tested. Similarly to the equivalence tests, the ergodicity test of [20] solely requires that the process in question is finitary. Therefore this efficient ergodicity test equally applies for quantum random walks.

\subsection{Conclusive Remarks}

We have presented a polynomial-time algorithm by which to efficiently test both hidden Markov processes and quantum random walks for equivalence. Previous solutions available for hidden Markov processes had runtime exponential in the number of hidden states. To test equivalence for quantum random walks, that is random walk models to be emulated on quantum computers, is relevant for the same reasons that apply for hidden Markov processes. An algorithm for testing equivalence for quantum random walks had not been available before. Note that the algorithm presented here is easy to implement and, in particular for hidden Markov processes, only requires invocation of well-known standard routines. Future directions are to explore how to efficiently test for similarity of hidden Markov processes and quantum random walks where similarity is measured in terms of approximate equivalence. Such tests have traditionally been of great practical interest.

\section{References}

1. D. Aharonov, A. Ambainis, J. Kempe, U. Vazirani: "Quantum walks on graphs", Proc. of 33rd ACM STOC, New York, pp. 50-59, 2001.

2. A. Ambainis: "Quantum search algorithms", SIGACT News, vol. 35(2), pp. 22-35, 2004.

3. D. Blackwell and L. Koopmans: "On the identifiability problem for functions of finite Markov chains", Annals of Mathematical Statistics, vol. 28, pp. 1011-1015, 1957.

4. M.A. Nielsen and I.L. Chuang: "Quantum Computation and Quantum Information", Cambridge University Press, Cambridge, UK, 2000.

5. P. Dupont, F. Denis and Y. Esposito: "Links between probabilistic automata and hidden Markov models: probability distributions, learning models and induction algorithms", Pattern Recognition, vol. 38, pp. 1349-1371, 2005.

6. Durbin, Eddy, Krogh: "Biological Sequence Analysis" Cambridge University Press, 1998 (XXX: check)

7. Y. Ephraim and N. Merhav: "Hidden Markov Processes", IEEE Transactions on Information Theory, vol. 48, 1518-1569, 2002.

8. U. Faigle and A. Schoenhuth: "Asymptotic mean stationarity of sources with finite evolution dimension", IEEE Transactions on Information Theory, vol. 53, 2342-2348, 2007.

9. U. Faigle and A. Schoenhuth: "Discrete Quantum Markov Chains”, Preprint 2010, submitted.

10. D. K. Faddeev and V. N. Faddeeva: "Computational Methods of Linear Algebra", Freeman, San Francisco, 1963.

11. E.J. Gilbert: "On the identifiability problem for functions of finite Markov chains", Annals of Mathematical Statistics, vol. 30, pp. 688-697, 1959. 
12. A. Heller: "On stochastic processes derived from Markov chains", Annals of Mathematical Statistics, vol. 36, pp. 1286-1291, 1965.

13. H. Ito, S.-I. Amari and K. Kobayashi: "Identifiability of hidden Markov information sources and their minimum degrees of freedom”, IEEE Trans. Inf. Theory, vol. 38(2), pp. 324-333, 1992.

14. H. Jäger: "Observable operator models for discrete stochastic time series", Neural Computation, vol. 12(6), pp. 1371-1398, 2000.

15. J. Kempe: "Quantum random walks hit exponentially faster", Probability Theory and Related Fields, vol. 133(2), pp. 215-235, 2005.

16. F.L. Marquezino, R. Portugal, G. Abal and R. Donangelo: "Mixing times in quantum walks on the hypercube", Phys. Rev. A, 77, 042312, 2008.

17. Y. Ephraim, N. Merhav, "Hidden Markov processes", IEEE Trans. on Information Theory, vol. 48(6), pp. 1518-1569, 2002.

18. R. Pintelon, J. Schoukens, "System Identification”, IEEE Press, Piscataway, NJ, 2001.

19. L.R. Rabiner: "A tutorial on hidden Markov models and selected applications in speech recognition", Proceedings of the IEEE, vol. 77, pp. 257-286, 1989.

20. A. Schönhuth, H. Jaeger: "Characterization of ergodic hidden Markov sources", IEEE Transactions on Information Theory, vol. 55, pp. 2107-2118, 2009.

21. W.-G. Tzeng: "A polynomial-time algorithm for the equivalence of probabilistic automata", SIAM Journal of Computing, vol. 21, pp. 216-227, 1992. 